\title{
Isolation, Identification, and In Vitro Antifungal Susceptibility Testing of Dermatophytes from Clinical Samples at Sohag University Hospital in Egypt
}

\author{
Mona Fattouh Mohamed Shalaby ${ }^{1}$, Asmaa Nasr El-din ${ }^{1}$, Mohammed Abu El-Hamd ${ }^{2}$
}

${ }^{1}$ Department of Medical Microbiology and Immunology, Faculty of Medicine, Sohag University, Egypt

${ }^{2}$ Department of Dermatology, Venereology and Andrology, Faculty of Medicine, Sohag University, Egypt

\section{Type of article: Original}

\begin{abstract}
Aim: The objective of this study was to isolate, identify, and explore the in-vitro antifungal susceptibility pattern of dermatophytes isolated from clinically suspected cases of dermatophytosis (tinea infections) attending the Dermatology Outpatient Clinic.

Methods: This study was conducted at Sohag University Hospital from December 2014 to December 2015. Clinical samples (e.g., skin scrapings and hair stumps) were collected under aseptic precautions. The identification of dermatophytes was performed through microscopic examination using $10 \%$ potassium hydroxide (KOH) with $40 \%$ dimethyl sulphoxide (DMSO) mounts and culture on Sabouraud dextrose agar (SDA) and on Dermasel agar base media, both supplemented with chloramphenicol and cycloheximide. All dermatophytes isolates were subjected to antifungal susceptibility testing using the agar-based disk diffusion (ABDD) method against Clotrimazole, Miconazole, Fluconazole, and Griseofulvin. Data were analyzed via SPSS 16, using Chi square and a screening test (cross-tabulation method).

Results: A total of 110 patients of dermatophytosis were studied. The patients were clinically diagnosed and mycologically confirmed as having tinea capitis (49), tinea corporis (30), tinea pedis (16), tinea cruris (9), or tinea barbae (6). The dermatophytes isolates belonged to 4 species: Microsporum canis 58 (52.7\%), Microsporum gypseum 23 (20.9\%), Trichophyton mentagrophytes 18 (16.4\%), and Microsporum audouinii $11(10 \%)$. The most effective antifungal drugs tested were Clotrimazole, followed by Miconazole $(95.5 \%$ and $84.5 \%$ of isolates were susceptible, respectively).

Conclusion: Every patient with a tinea infection should be properly studied for a mycological examination and should be treated accordingly. Dermasel agar is more useful as an identification medium in the isolation of dermatophytes. The ABDD method appears to be a simple, cost-effective, and promising method for the evaluation of antifungal susceptibility of dermatophytes.
\end{abstract}

Keywords: Dermatophytosis; Dermasel agar; Disk diffusion; Antifungal agents

\section{Introduction}

Dermatophytes are a group of closely related filamentous fungi able to damage and utilize keratin found in the skin, hair, and nails (1). They are classified into three genera: Microsporum, Trichophyton, and Epidermophyton. Dermatophytosis is an infection produced by dermatophytic fungi in the keratinized tissues (2). These cutaneous mycoses affect $20 \%$ to $25 \%$ of the world's population (3). Clinically, dermatophytosis can be classified depending on the site involved. These include Tinea capitis (scalp), Tinea corporis (non-hairy skin of the body), Tinea ungium (nail infection), Tinea cruris (groin), Tinea pedis (athlete's foot), and Tinea barbae (bearded areas of the face and neck) (4). The dermatophytosis transfers from animal (zoophilic dermatophytes) and soil (geophilic dermatophytes) to man or through direct infection by personal contact (anthropophilic dermatophytes) (5). The hot and humid climate in tropical and subtropical countries like Egypt makes dermatophytosis a very common fungal skin infection. Generally, they are superficial infections, but in immunocompromised patients they are experienced as a

\section{Corresponding author:}

Assistant Professor Dr. Mona Fattouh Mohamed Shalaby, Department of Medical Microbiology and Immunology, Faculty of Medicine, Sohag University, Egypt. Tel: +201009222054, E-mail: monarahman2002@yahoo.co.uk Received: March 22, 2016, Accepted: May 23, 2016, Published: June 2016 iThenticate screening: May 23, 2016, English editing: May 28, 2016, Quality control: June 02, 2016

(C) 2016 The Authors. This is an open access article under the terms of the Creative Commons Attribution-NonCommercialNoDerivs License, which permits use and distribution in any medium, provided the original work is properly cited, the use is non-commercial and no modifications or adaptations are made. 
disseminated disease. The gross appearance of the lesion includes an outer ring of active, progressing infection with central healing within the ring accompanied with itching, redness, scaling, or fissuring of the skin (6). If the infection involves the scalp, areas of scaling with dull gray hair stumps up to a highly inflammatory reaction with folliculitis, kerion formation, and alopecia (7). More aggressive infections may lead to an abscess or cellulitis. Areas infected by dermatophytes may become secondarily infected by bacteria. Symptoms typically appear between 4 and 14 days following exposure (8). The diagnosis of a dermatophytic infection is mostly done clinically, but often confused with other skin infections due to the topical application of steroid ointments and creams, leading to further misdiagnosis and mismanagement (9). Hence, there arises the need for the correct, efficient, and rapid laboratory diagnosis of dermatophytes. The laboratory identification of dermatophytes is typically based on the macroscopic observation of colony morphology (pigmentation, growth rate, texture, etc.) grown on selective media, followed by a microscopic examination of conidia (10). Another important point to consider is that resistance to antifungals has started appearing in dermatophytes. The establishment of a reference antifungal susceptibility testing method may allow the clinician to select the appropriate therapy for the treatment of infections caused by dermatophytic fungi. The evaluation of in-vitro susceptibility testing has been hampered due to the lack of reliable in-vitro techniques for testing antifungal agents against dermatophytes. The agar-based disk diffusion (ABDD) susceptibility method for dermatophytes is quick, easy, and inexpensive and does not require specialized equipment, making it a good option (11). However, data on disk diffusion methods for dermatophytes are scarce (12). Given this background, our study focused primarily on how to isolate, identify, and detect the in-vitro sensitivity pattern of various dermatophytes isolates, from clinically suspected cases of dermatophytosis to the most commonly used antifungal agents, using the ABDD susceptibility method.

\section{Material and Methods}

\subsection{Study design and patients}

This study was carried out in the Department of Medical Microbiology \& Immunology and Department of Dermatology, Venereology \& Andrology, Faculty of Medicine, Sohag University, Egypt, between December 2014 and December 2015. Clinically suspected cases of dermatophytosis attending the Dermatology Outpatient Clinic at Sohag University Hospital were included in the study, irrespective of their age or gender. Patients who received topical or systemic antifungal treatment in the four weeks preceding sampling were excluded from this study. Patients' personal history, including age, sex, occupation, and other demographic profile, was noted. After the detailed history, a clinical examination of patients was conducted in good light, including the site of the lesion, number of lesions, types, and presence of an inflammatory margin. Samples were collected after obtaining informed oral consent from the patients. The study was approved by the ethical committee of Sohag Faculty of Medicine.

\subsection{Specimen collection}

Suspected lesions were cleaned with $70 \%$ ethyl alcohol to remove any dirt and contaminating bacteria. Skin scales and crusts were collected from the erythematous, peripheral, actively growing margins of the lesions by scraping across the inflamed margin of the lesion into the apparently healthy tissue using the blunt edge of a sterile surgical blade onto clean glass slides. Hair specimens were collected by using epilating forceps to pluck along the base of the hair shaft, and scales were scraped from the surface using the blunt edge of a sterile surgical blade. The cutting of hair was avoided as the infection is usually confined to the root, very near the scalp's surface. Specimens were collected and sealed in sterile dry Petri dishes; they were labeled with the patient's name, age, sex, date of collection, and site of infection and subsequently brought to the laboratory for mycological examination. The samples were divided into two portions: one for microscopic examination and one for culture.

\subsection{Microscopic examination and fungal culture}

For direct microscopy, the samples collected were screened for the presence of fungal elements using a $10 \% \mathrm{KOH}$ with 40\% Dimethyle sulphoxide (DMSO) mount mixed in equal proportion. Two to three drops of the $\mathrm{KOH}+\mathrm{DMSO}$ mixture were kept on a clean, grease-free glass slide. The sample (skin scraping or hair plucking) was placed in the $\mathrm{KOH}+\mathrm{DMSO}$ drops on the slide, and a clean cover slip was placed on the sample and pressed to prevent the formation of air bubbles. The sample was kept in $\mathrm{KOH}+\mathrm{DMSO}$ and then observed after 5-8 minutes. DMSO increases the sensitivity of the preparation and softens keratin more quickly than $\mathrm{KOH}$ alone in the absence of heat. Each slide was thoroughly examined under low power (10X) and high power (40X) magnification for the presence of hyphae and/or arthroconidia. On the surface of the shaft of infected hairs, the mosaic arrangement of spores was seen (ectothrix infection) or hyphal fragments and arthroconidia was seen internally (endothrix infection) (Figure 1). After a direct microscopic examination, irrespective of the demonstration of fungal elements, hair or skin scraping specimens were inoculated in two sets of Petri dishes: one in a Sabouraud dextrose agar (SDA) base (Oxoid, UK) 
and the other in a Dermasel agar base (Oxoid, UK), both supplemented with chloramphenicol (acts as a broad spectrum antibiotic, which inhibits a wide range of gram-positive and gram-negative bacteria) and cycloheximide (to inhibit saprophytic fungi). To prepare selective media, one vial of Dermasel Selective Supplement SR0075 (Oxoid, UK) was added, after being reconstituted as directed in the product insert, to each $500 \mathrm{ml}$ of medium, resulting in $0.4 \mathrm{~g} / \mathrm{l}$ of cycloheximide and $0.05 \mathrm{~g} / \mathrm{l}$ of chloramphenicol. Cultures were incubated aerobically at room temperature $\left(25^{\circ} \mathrm{C}\right)$ for up to 4 weeks. Positive cultures were examined both macroscopically (color of the surface and reverse, topography, and texture) and microscopically (two types of conidia were formed by dermatophytes: small unicellular microconidia and larger septate macroconidia) for species identification. In the absence of any growth after 4 weeks, the culture was considered negative (Figures 2 and 3).

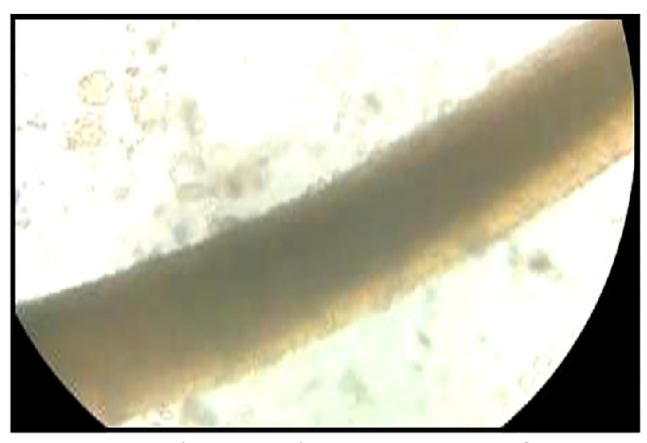

Figure 1. Microscopic appearance of $10 \% \mathrm{KOH} / 40 \%$ DMSO mount of hair, showing Ectothrix hair invasion with the formation of arthroconidia on the outside of the hair shaft; the cuticle of the hair was destroyed.
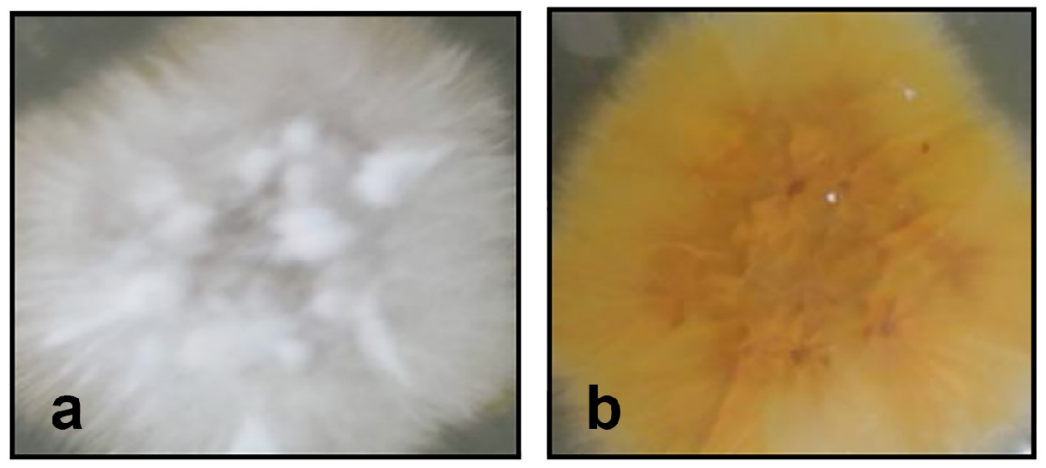

Figure 2. Macroscopic appearance of culture of Microsporum canis on Dermasel agar base supplemented with chloramphenicol and cycloheximide: a) flat spreading white to cream colored with dense cottony surface and b) bright golden yellow reverse pigment.

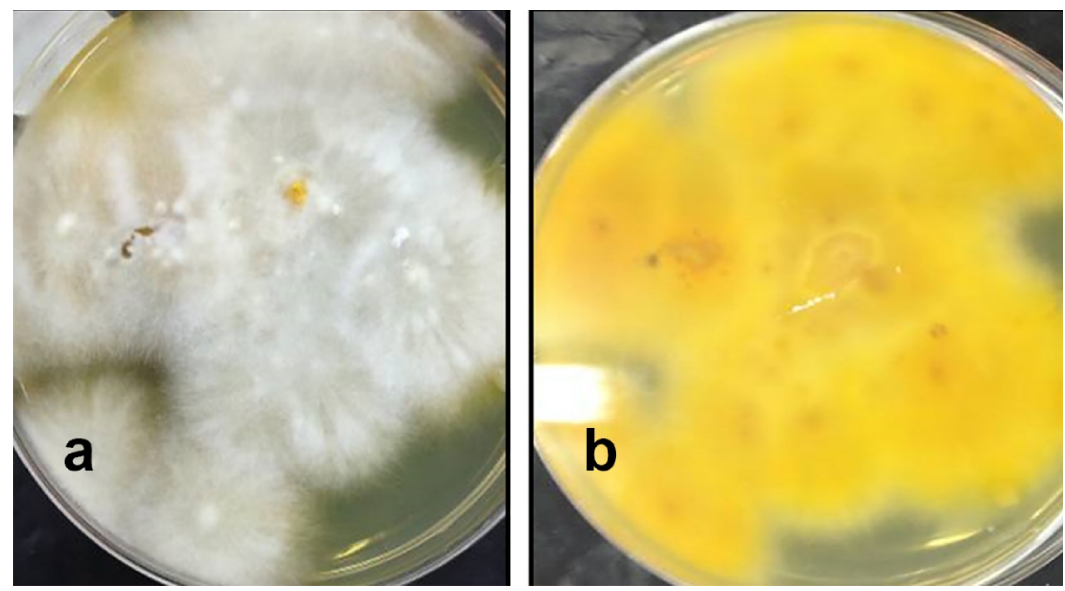

Figure 3. Macroscopic appearance of culture of Microsporum gypseum on Dermasel agar base supplemented with chloramphenicol and cycloheximide: a) flat, granular, with a deep cream to pale cinnamon-colored surface and b) yellow brown reverse pigment. 
To obtain a sample for microscopic fungal identification, a small piece of clear acetate tape was gently touched to the surface of the fungal colony, and then the tape was carefully applied to a glass slide over of a drop of methylene blue stain; the slide was examined under $10 \mathrm{X}$ and $40 \mathrm{X}$ magnification to identify the characteristic dermatophyte conidia and fungal hyphae (Figures 4-7).
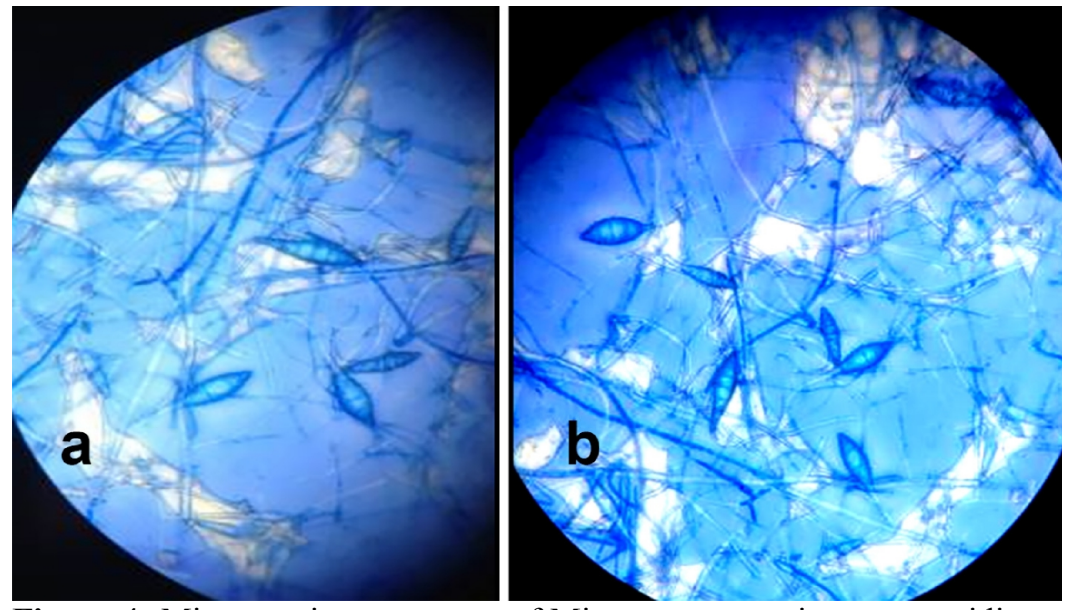

Figure 4: Microscopic appearance of Microsporum canis macroconidia; spindle shaped with 5-15 cells with rough surface, a thick outer wall, and a terminal knop.

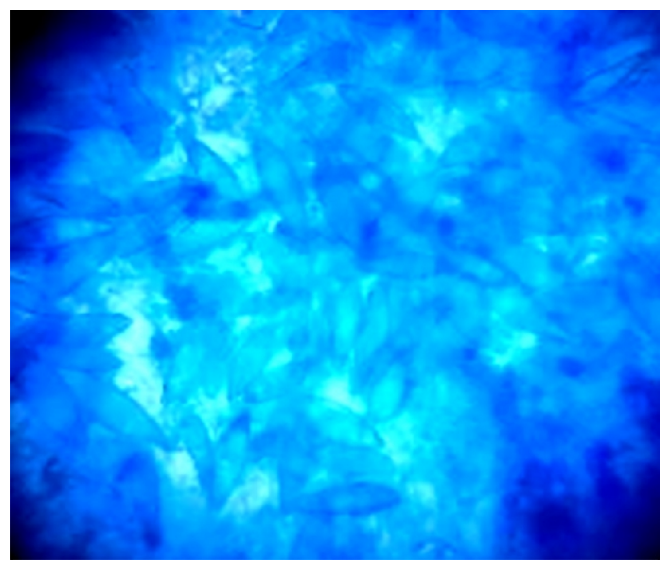

Figure 5. Microscopic appearance of Microsporum gypseum macroconidia; ellipsoidal and thin walled.

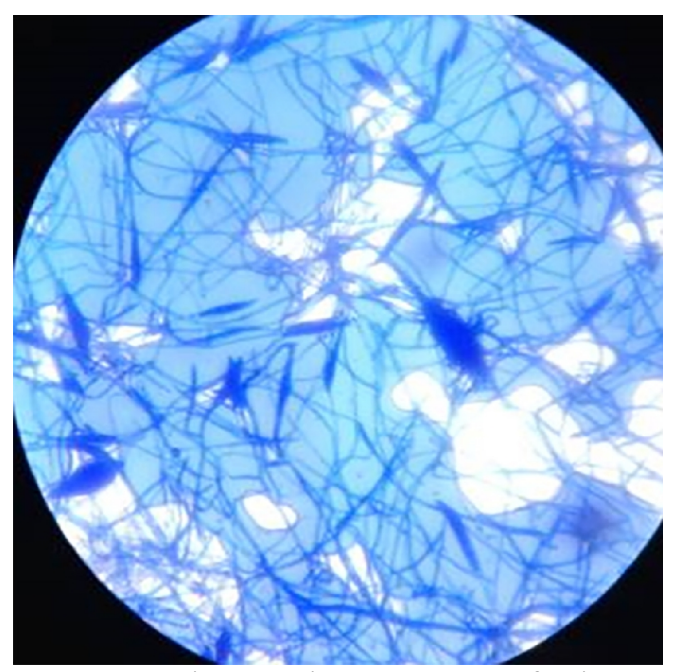

Figure 6. Microscopic appearance of Microsporum audouinii macroconidia. Some are long and thin; others are the typical spindle shape; some show a slight constriction along their length near the middle of the macroconidia. 


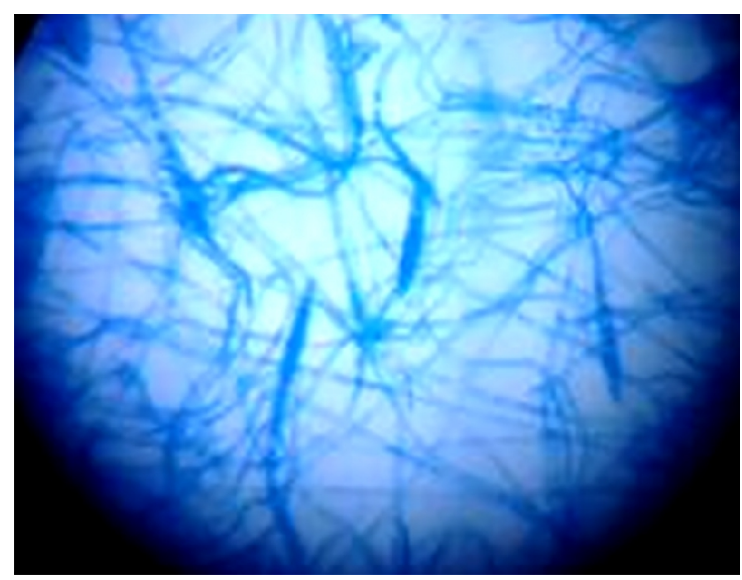

Figure 7. Microscopic appearance of Trichophyton mentagrophytes showing thin and pencil-shaped macroconidia present in small numbers.

\subsection{Antifungal susceptibility testing:}

ABDD antifungal susceptibility testing was performed using four antifungal agents: Clotrimazole $(50 \mu \mathrm{g})$, Miconazole $(10 \mu \mathrm{g})$, Fluconazole $(25 \mu \mathrm{g})$, and Griseofulvin $(10 \mu \mathrm{g})$ (Liofilchem diagnostic, Italy). Dermatophytes colonies were probed from Dermasel agar cultures using the tip of a sterile Pasteur pipette to obtain a mixture of mycelium and conidia and mixed in $1 \mathrm{ml}$ distilled water; the mixture was suspended in sterile tubes and allowed to sediment for 30 minutes. Swabs dipped into the inocula suspensions were streaked evenly over the surface of Mueller-Hinton (MH) agar (Oxoid, UK) plates. Lids were left ajar for 3 minutes in a laminar flow cabinet to allow for any excess surface moisture to be absorbed into the agar before the drug-impregnated disks were applied. Disks containing the test agents were applied to the surfaces of inoculated plates, and the plates were incubated at $25^{\circ} \mathrm{C}$ for 5 to 10 days. When growth took place, the size of the zones of inhibition around the disks was measured and recorded. Criteria of susceptibility and resistance of antifungal agents were measured according to Pakshir et al. (13) (Table 1, Figure 8).

Table 1. Criteria of susceptibility and resistance of antifungal agents

\begin{tabular}{|l|l|l|l|l|}
\hline Antifungal drug & \multirow{2}{*}{ Potency } & \multicolumn{3}{|l|}{ Zone Diameter in mm } \\
\cline { 2 - 5 } & & Sensitive & Intermediate & Resistant \\
\hline Clotrimazole (CLO) & $50 \mu \mathrm{g}$ & $\geq 20$ & $19-12$ & $\leq 11$ \\
\hline Miconazole (MCL) & $10 \mu \mathrm{g}$ & $\geq 20$ & $19-12$ & $\leq 11$ \\
\hline Fluconazole (FLU) & $25 \mu \mathrm{g}$ & $\geq 22$ & $21-15$ & $\leq 14$ \\
\hline Griseofulvin (AGF) & $10 \mu \mathrm{g}$ & $\geq 10$ & - & No zone \\
\hline
\end{tabular}

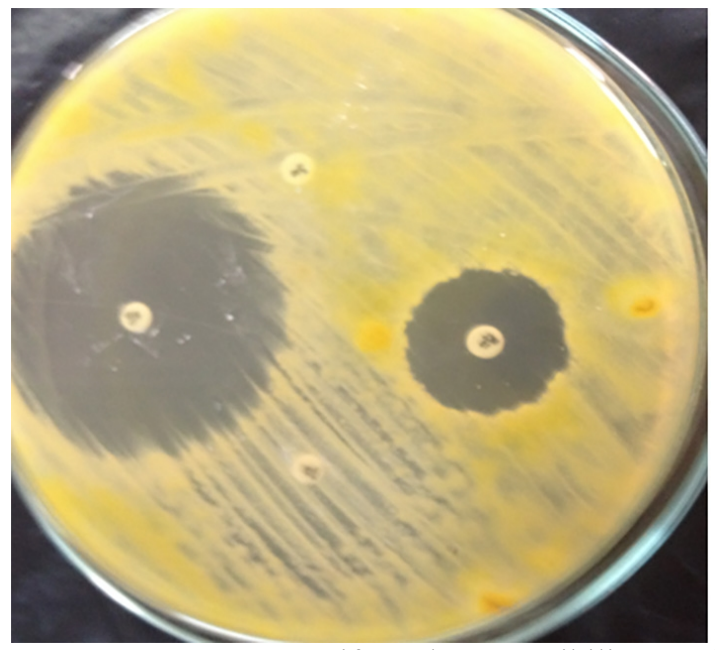

Figure 8. ABDD antifungal susceptibility testing of dermatophytes showing resistance to Fluconazole and Griseofulvin. 


\subsection{Statistical analysis}

Data were analyzed using SPSS version 16 (SPSS Inc., Chicago, Illinois, USA). Qualitative data were expressed as frequency and percentage, and quantitative data were expressed as mean \pm standard deviation of the mean (SD). A Chi square test was used to compare frequencies of qualitative data, and sensitivity and specificity were calculated using a screening test (cross-tabulation method).

\section{Results}

A total of 135 clinically suspected cases of skin and hair dermatophytosis were included in the study. Dermatophytes were demonstrated in 110 cases (81.5\%)-71 (64.5\%) males and 39 (35.5\%) females-through direct microscopy and/or culture. The age of the study group ranged from 5 to 56 years (mean age 30.5 years), with the maximum number of patients falling in the 16-30 years age group (32.7\%), followed by 5-15 years (30\%). A considerable number of the patients (39.1\%) were from rural areas and engaged in occupations related to agriculture, and $22.7 \%$ of patients were students. The patients were clinically diagnosed and mycologically confirmed to have tinea capitis (49 cases; 44.5\%) presented with hair loss, scaling, and/or black dots (Figure 9); tinea corporis (30 cases; $27.3 \%$ ) presented with pruritic, characteristic annular progressive scaly plaque with a burning sensation and healed center (Figure 10); tinea pedis (16 cases; 14.5\%) presented with moist peeling irritable skin in the cleft between the toes (Figure 11); tinea cruris (9 cases; $8.2 \%$ ) presented with pruritus and erythematous patches with sharp border in the groin; and tinea barbae (6 cases; $5.5 \%$ ) presented with red plaques, pustules, and hair loss in moustache and beard (Table 2).

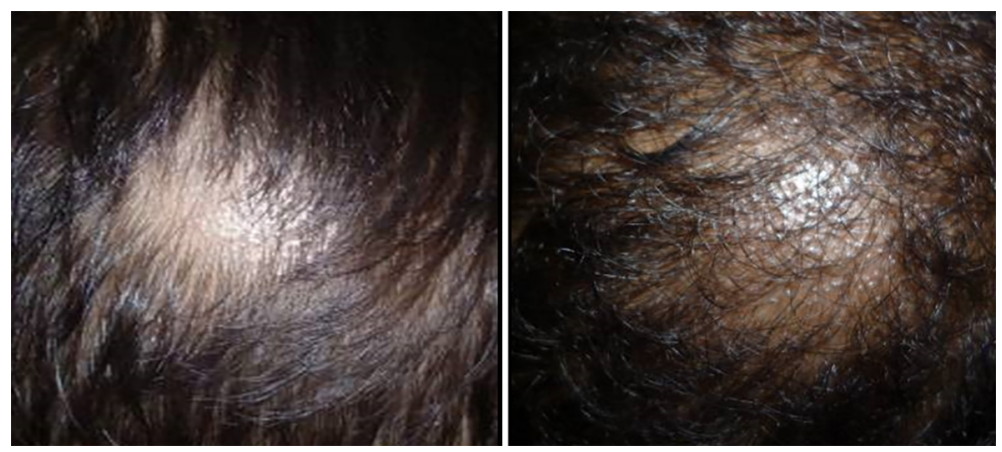

Figure 9. Tinea capitis showing circular lesions with black dot alopecia

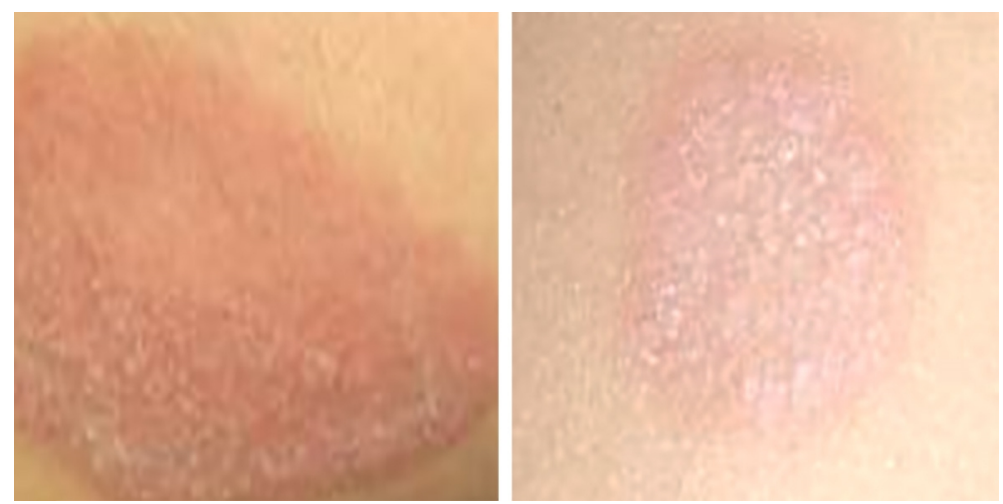

Figure 10. Tinea corporis showing circular erythematous scaly lesions with advancing margin on the abdomen.

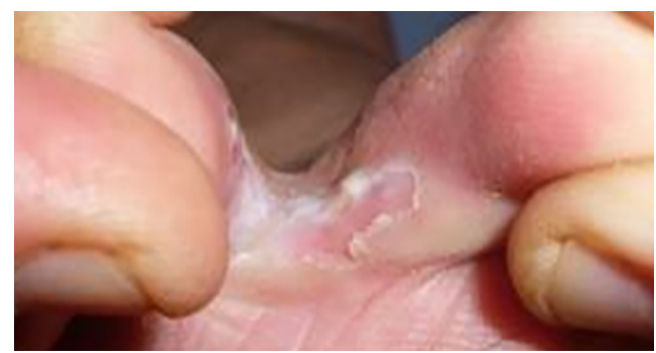

Figure 11. Tinea pedis showing lesions between the toes. 
Table 2. Distribution of clinical types of dermatophytosis in relation to the age and sex of patients

\begin{tabular}{|l|l|l|l|l|l|l|l|}
\hline \multirow{2}{*}{ Type of infection } & \multicolumn{4}{|l|}{ Age groups (year) } & \multicolumn{2}{l|}{ Sex } & Total \\
\cline { 2 - 8 } & $5-15$ & $16-30$ & $31-45$ & $46-65$ & Male & Female & \\
\hline Tinea capitis & 29 & 13 & 4 & 3 & $31(43.7 \%)$ & $18(46.2 \%)$ & $49(44.5 \%)$ \\
\hline Tinea corporis & 4 & 12 & 9 & 5 & $20(28.2 \%)$ & $10(25.6 \%)$ & $30(27.3 \%)$ \\
\hline Tinea pedis & - & 9 & 5 & 2 & $10(14.1 \%)$ & $6(15.4 \%)$ & $16(14.5 \%)$ \\
\hline Tinea cruris & - & 1 & 6 & 2 & $4(5.6 \%)$ & $5(12.8 \%)$ & $9(8.2 \%)$ \\
\hline Tinea barbae & - & 1 & 4 & 1 & $6(8.5 \%)$ & $-(0 \%)$ & $6(5.5 \%)$ \\
\hline
\end{tabular}

The Dermasel agar culture was able to detect 110 cases $(81.5 \%)$ from the 135 clinically suspected cases of skin and hair dermatophytosis whereas the culture on SDA was able to detect 107 cases $(79.3 \%)$. No statistically significant difference was evident between the two media $(\mathrm{P}<0.05)$ for primary isolation of dermatophytes from the clinical samples. Ninety-seven cases $(71.9 \%)$ were positive in both the microscopic examination and culture, 13 cases (9.6\%) were negative in microscopy but culture positive, 6 cases $(4.4 \%)$ were positive in microscopy and negative in culture, and 19 cases (14.1\%) were negative in both microscopy and culture. Thus, the culture is more accurately confirmatory than the microscopic mount. The sensitivity and specificity of the $10 \% \mathrm{KOH} / 40 \%$ DMSO mount examination was calculated as $88.2 \%$ and $76 \%$, respectively, when compared with reference standard culture results (Table 3). A total of 110 isolates of dermatophytes was identified by macroscopic and microscopic morphological characteristics. The isolates belonged to two genera: Microsporum (92 cases) - the most prevalent cause of dermatophytosis in our study, accounting for $83.6 \%$ of isolates - and Trichophyton (18 cases; $16.4 \%)$. The isolates included four species: (M. canis \{zoophilic\} 58 (52.7\%), M. gypseum \{geophilic\} 23 (20.9\%), M. audouinii \{anthropophilic\} $11(10 \%)$, and T. mentagrophytes \{zoophilic\} $18(16.4 \%)$ (Table 4). The results of the susceptibility testing to antifungal drugs were as follows: Clotrimazole-105 $(95.5 \%)$ susceptible and $5(4.5 \%)$ of intermediate susceptibility; Miconazole-93 (84.5\%) susceptible, 12 (10.9\%) of intermediate susceptibility and 5 (4.5\%) resistant; Griseofulvin-51 (46.4\%) susceptible and 59 (53.6\%) resistant; and Fluconazole-104 (94.5\%) resistant and $6(5.5 \%)$ of intermediate susceptibility. Based on these data, Clotrimazole was the most effective antifungal drug and Fluconazole had the poorest activity (Table 5). In general, different species of dermatophytes showed similar patterns of susceptibility to antifungal agents tested (Table 6).

Table 3. Correlation between findings of $10 \% \mathrm{KOH} / 40 \%$ DMSO mount and culture

\begin{tabular}{|l|l|l|l|}
\hline \multirow{2}{*}{ Results of culture } & Results of KOH mount \\
\cline { 2 - 4 } & KOH mount positive & KOH mount negative & Total \\
\hline Culture positive & 97 & 13 & 110 \\
\hline Culture negative & 6 & 19 & 25 \\
\hline Total & 103 & 32 & 135 \\
\hline
\end{tabular}

Table 4. Isolated species of dermatophytes in relation to clinical types of the disease

\begin{tabular}{|c|c|c|c|c|}
\hline \multirow{2}{*}{$\begin{array}{l}\text { Clinical } \\
\text { types }\end{array}$} & \multicolumn{4}{|c|}{ Species of dermatophytes; $\mathrm{n}(\%)$} \\
\hline & $\begin{array}{l}\text { Microsporum } \\
\text { canis }\end{array}$ & $\begin{array}{l}\text { Microsporum } \\
\text { gypseum }\end{array}$ & $\begin{array}{l}\text { Trichphyton } \\
\text { mentagrophytes }\end{array}$ & $\begin{array}{l}\text { Microsporum } \\
\text { audouinii }\end{array}$ \\
\hline T. capitis & $28(25.5)$ & $12(10.1)$ & $4(3.6)$ & $5(4.5)$ \\
\hline T. corporis & $13(11.8)$ & $5(4.5)$ & $9(8.2)$ & $3(2.7)$ \\
\hline T. pedis & $6(5.4)$ & $4(3.6)$ & $4(3.6)$ & $2(1.8)$ \\
\hline T. cruris & $6(5.5)$ & $2(1.8)$ & $-(0)$ & $1(0.9)$ \\
\hline T. barbae & $5(4.5)$ & $-(0)$ & $1(0.9)$ & $-(0)$ \\
\hline
\end{tabular}

Table 5. Antifungal susceptibility pattern of the isolated dermatophytes species

\begin{tabular}{|l|l|l|l|}
\hline \multirow{2}{*}{ Antifungal drug } & \multicolumn{3}{|l|}{ Susceptibility pattern; n (\%) } \\
\cline { 2 - 4 } & Susceptible & Intermediate susceptibility & Resistant \\
\hline Clotrimazole & $105(95.5)$ & $5(4.5)$ & $-(0)$ \\
\hline Miconazole & $93(84.5)$ & $12(10.9)$ & $5(4.5)$ \\
\hline Griseofulvin & $51(46.4)$ & - & $59(53.6)$ \\
\hline Fluconazole & $-(0)$ & $6(5.5)$ & $104(94.5)$ \\
\hline
\end{tabular}


Table 6. Distribution of antifungal susceptibility pattern of different dermatophyte species

\begin{tabular}{|l|l|l|l|l|l|l|l|l|}
\hline \multirow{2}{*}{$\begin{array}{l}\text { The tested antifungal } \\
\text { drug }\end{array}$} & \multicolumn{2}{l}{$\begin{array}{l}\text { Microsporum } \\
\text { canis }\end{array}$} & \multicolumn{2}{l|l}{$\begin{array}{l}\text { Microsporum } \\
\text { gypseum }\end{array}$} & \multicolumn{2}{l}{$\begin{array}{l}\text { T. } \\
\text { mentagrophytes }\end{array}$} & \multicolumn{2}{l}{$\begin{array}{l}\text { Microsporum } \\
\text { audouinii }\end{array}$} \\
\cline { 2 - 12 } & $\mathrm{n}$ & $\%$ & $\mathrm{n}$ & $\%$ & $\mathrm{n}$ & $\%$ & $\mathrm{n}$ & $\%$ \\
\hline Clotrimazole & 56 & 96.6 & 22 & 95.6 & 17 & 94.4 & 10 & 90.9 \\
\hline Miconazole & 50 & 86.2 & 19 & 82.6 & 15 & 83.3 & 9 & 81.8 \\
\hline Griseofulvin & 27 & 46.6 & 11 & 47.8 & 8 & 44.4 & 5 & 45.5 \\
\hline Fluconazole & - & - & - & - & - & - & - & - \\
\hline
\end{tabular}

\section{Discussion}

Dermatophytosis is a major public health problem in tropical and subtropical countries, like Egypt, yet remains unresolved. Therefore, it is essential that good laboratory methods are available for the rapid and precise identification of the dermatophytes involved in order to apply appropriate treatment and prevention measures. In the present study, 110 clinically suspected cases of tinea infections were examined over a period of 12 months, among which the most common tinea infections were T. capitis (49 cases), followed by T. corporis (30), T. pedis (16), T. cruris (9), and T. barbae (6 cases). This finding concurred with Omar's (14) results, which emphasized that T. capitis is considered the major type of fungal infection in Egypt. Our results also agreed with those of Achterman and White (15), who reported that - although dermatophytosis is found throughout the world - developed countries have high rates of tinea pedis and onychomycosis whereas developing countries, like Egypt, have high rates of tinea capitis. In the present study, most of the infections were seen in the younger age group (5-30 years), which may be due to increased physical activity and increased opportunity for exposure. Indeed, Madhavi et al. (2011) found that tinea infections were more common in the 16- to 45-year-old age group. In our study, a higher incidence of tinea infection of the capitis variety in particular in school-going children, resulting in increased transmission between them, would be due to increased contact, overcrowding in classrooms (a significant problem in Egypt), a lack of awareness and apathy to personal hygiene, the sharing of personal items, and exposure to soil and even animals on playgrounds. A higher incidence of dermatophytosis was seen in males than in females, which supports Sumathi et al.'s (17) findings. Male predominance may be due to increased outdoor physical activities and increased sweating, which create a favorable environment for fungal infections, as well as a greater opportunity for exposure to infection than females. In our study, the most common genus of dermatophytes detected was Microsporum, followed by Trichophyton. However, Jha et al. (18) found that the most common genus was Trichophyton, followed by Epidermophyton. In the present study; M. canis (zoophilic dermatophytes) was the most common isolate (58 cases; $52.7 \%$ ), followed by M. gypseum (geophilic dermatophytes; 23 cases; 20.9\%), T. mentagrophytes (zoophilic dermatophytes; 18 cases; 16.4\%), which could be due to patients' interaction with soil and domestic animals, and finally M. audouinii (anthropophilic dermatophytes; 11 cases; 10\%). This finding did not agree with that of Kannan et al. (19), who reported that Trichophyton rubrum (73\%) was the most common isolate, followed by Trichophyton mentagrophytes (17\%), Trichophyton violaceum, and finally Epidermophyton floccosum. The differences between our study and previous studies stem from variations in patients' environments and lifestyles. In the current study, zoophilic fungi represent $69.1 \%$ of the isolated dermatophytes (M. canis $52.7 \%$ and T. mentagrophytes $16.4 \%$ ). This result did not differ greatly from previously reported findings in Egypt (20), which could be due to the fact that a considerable number of our patients $(39.1 \%)$ were from rural areas and engaged in agricultural occupations. This finding concurred with Emele and Oyeka's (21) findings, which indicated that purely rural agricultural communities, coupled with the associated crowded and unhygienic rural lifestyles and low economic conditions, might have enhanced the spread of the disease among the population in these regions. Although our results demonstrated a predominance of zoophilic dermatophytes, other categories were also detected, which was similar to Nweze and Okafor's (22) findings. These researchers concluded that species of dermatophytes causing different types of tinea vary from country to country and also change with time, geography, environment, climate, occupation, and lifestyles. In the present study, aqueous potassium hydroxide $(\mathrm{KOH})$ was used as a clearing agent for the direct demonstration of fungi in skin or hair scrapings (23), but the addition of dimethyl sulphoxide, as described by Rebell and Taplin (24), was found to be a better preparation over plain $\mathrm{KOH}$. The addition of DMSO allows for the rapid clearing of keratin and almost immediate examination of the sample without a warming of the slide (25). It also prevents the rapid drying of the fluid and, thus, is a better option. Our findings suggested that a $10 \% \mathrm{KOH} / 40 \%$ DMSO test can be used for the diagnosis of dermatophytes infections in remote conditions, where a rapid and lowcost diagnosis is required. However, false positive cases were recorded in our study: 6 cases $(4.4 \%)$ were positive by microscopy but negative by culture. In addition, false negative cases were recorded: 13 cases $(9.6 \%)$ were negative by microscopy but positive by culture. This variation could be due to the non-viability of fungal elements in some cases, inadequacy in sampling due to very small lesions, and possible non-reported partial treatment with antifungal 
agents. On the other hand, Tampieri (26) reported that it seems difficult to rely on results of direct microscopy with $\mathrm{KOH}$ to establish the diagnosis of fungal infection as it could not detect the characteristic morphology of the three genera and it lacks sufficient sensitivity, although it is highly efficient as a screening technique before therapy is initiated because of the expense, duration, and potential adverse effects of the treatment. Given that culture is the gold standard for the isolation and identification of dermatophytes, the sensitivity and specificity of the $10 \%$ $\mathrm{KOH} / 40 \%$ DMSO mount examination in our study was calculated as $88.2 \%$ and $76 \%$, respectively, when compared with the reference standard culture results. This finding concurred with Girgis et al. (27), who found that KOH direct microscopy had a sensitivity of $88 \%$ and specificity of $74 \%$. Culture is considered the mainstay of diagnosis because it not only isolates the organism, but also allows for the identification of the etiologic agent, thereby allowing treatment to be tailored appropriately. In our study, two media were used for culture of samples; both the Dermasel agar and SDA were supplemented with chloramphenicol and cycloheximide. The two media proved to be technically good, with no statistically significant difference between them $(\mathrm{P}<0.05)$ for the primary isolation of dermatophytes from clinical samples. However, of much concern was our finding that the culture of dermatophytes on Dermasel agar was more specific with better topography, texture, color, and colony surface and reverse; macroconidia and microconidia are typical for the species when studied microscopically, and less growth of contaminant bacteria and saprophytic fungi is common. This elevates the importance of Dermasel agar as a selective medium for the isolation of dermatophytes and, thus, the treatment of infection. There were reports concerning the failure of topical, as well as systemic antimycotic treatment of dermatophyte infections (28), thereby making it essential to evaluate and standardize simple and reproducible in-vitro assays to determine the antifungal activity of various drugs against dermatophytes. Studies evaluating the in-vitro activity of antifungal agents are rare, particularly in filamentous fungi (29). The development of a standardized disk diffusion agar-based assay for determining the antifungal susceptibility of dermatophytes is desirable as it is very simple, easy to use, and economical, making it appropriate for use in routine clinical testing. In the present study, we tested the susceptibility pattern of 110 isolates of dermatophytes against 4 commonly used antifungal agents for the treatment of dermatophytosis - namely, Clotrimazole, Miconazole, Fluconazole, and Griseofulvin - using ABDD antifungal susceptibility testing. Nweze et al. (30) optimized the best growth medium to use in the disk diffusion assay and tested the Mueller-Hinton (MH) medium alone and supplemented with $2 \%$ glucose and $0.5 \mu \mathrm{g} / \mathrm{ml}$ methylene blue. Their data showed that the use of the MH medium alone resulted in clear inhibition zones for all strains tested in the study while supplementation with $2 \%$ glucose and $0.5 \mu \mathrm{g} / \mathrm{ml}$ methylene blue did not enhance the clarity in zone edge definition. Based on these data, we identified non-supplemented MH medium as an optimal medium. Another important variable that can affect the test is the type of inoculum preparation; many professionals, including CLSI guidelines, recommend the use of microconidia (30). Microconidia of the tested species present higher susceptibility to antifungal drugs than hyphal preparations (31), which may explain the low MIC or very large inhibition zone diameter (IZD) by several of them. Therefore, in our study, we used a mixture of hyphae and conidia. Inoculum size and incubation temperature may also affect the results of antifungal sensitivity testing. Fernández-Torres et al. (32) and Santos et al. (31) demonstrated that inoculum size does not affect the result. In our study, the inoculum used was prepared by taking dermatophytes colonies from Dermasel agar cultures using the tip of a sterile Pasteur pipette to obtain a mixture of mycelium and conidia that was then mixed in $1 \mathrm{ml}$ of distilled water. Santos et al. (31) concluded that temperature alone $\left(28^{\circ} \mathrm{C}\right.$ or $\left.35^{\circ} \mathrm{C}\right)$ does not significantly affect the results. We used an incubation temperature of $28^{\circ} \mathrm{C}$. Among the antifungal drugs tested, Clotrimazole was the most potent antifungal agent. Clotrimazole is one of the oldest antifungal drugs formulated as a topical for use against dermatophytosis. This antimycotic agent showed an excellent in-vitro potency against all the tested species, suggesting that it can be used to treat a majority of dermatophytic infections. Furthermore, in agreement with results published in previous studies, Fluconazole and Griseofulvin were found to be the least active among the evaluated antifungal agents $(33,34)$. In this study, Fluconazole had the poorest activity on isolates tested; as in most isolates, no inhibition zones were observed around the disks. Our data concurred with many studies indicating that Fluconazole was the least effective against dermatophytes (28). Our work suggests that in-vitro ABDD antifungal susceptibility testing is simple, is inexpensive, and does not require specialized equipment; thus, it can be used for the routine assessment of dermatophyte susceptibility to antifungal agents in the routine clinical laboratory. It allows for a comparison between different antifungal agents and may help optimize the therapy for treating patients with dermatophytosis. This study also provided new insights into some epidemiological profiles of dermatophytosis and transmission portals, which will be useful for the efficient future screening, management, reduction, and treatment of this disease, which is a public health problem in our locality. 


\section{Conclusions}

Dermatophytes infections are very common in our country, where the hot and humid climate in association with poor hygienic conditions play an important role in the growth of these fungi. Our study signified the importance of the mycological examination in the diagnosis of various dermatophytosis for their effective management. Dermasel agar can be used as a rapid screening medium for the isolation and identification of dermatophytes compared to SDA. The ABDD antifungal susceptibility testing for dermatophytes can be adapted for the routine assessment of dermatophyte resistance to antifungal agents and can aid the clinician in initiating prompt and appropriate antifungal therapy.

\section{Acknowledgments:}

We thank Sohag University for contributing to this research.

\section{Conflict of Interest:}

There is no conflict of interest to be declared.

\section{Authors' contributions:}

All authors contributed to this project and article equally. All authors read and approved the final manuscript.

\section{References:}

1) Alshawa K, Beretti JL, Lacroix C, Feuilhade M, Dauphin B, Quesne G, et al. Successful identification of clinical dermatophytes and Neoscytalidium species by matrix-assisted laser desorption ionization-time of flight mass spectrometry. J Clin Microbiol. 2012; 50(7): 2277-81. doi: 10.1128/JCM.06634-11. PMID: 22535981, PMCID: PMC3405581.

2) Grumbt M, Monod M, Yamada T, Hertweck C, Kunert J, Staib P. Keratin degradation by dermatophytes relies on cysteine dioxygenase and a sulfite efflux pump. J Invest Dermatol. 2013; 133(6): 1550-5. doi: 10.1038/jid.2013.41. PMID: 23353986.

3) Havlickova B, Czaika VA, Friedrich M. Epidemiological trends in skin mycoses worldwide. Mycoses. 2008; 51(4): 2-15. doi: 10.1111/j.1439-0507.2008.01606.x. PMID: 18783559.

4) Ananthanarayan R, Paniker CK. Medical mycology, Chapter 65.Textbook of Microbiology; 8th edition. Hyderabad, India: Universities Press Private Limited. 2009; 604-7.

5) Achterman RR, White TC. Dermatophyte virulence factors: Identifying and analyzing genes that may contribute to chronic or acute skin infections. Int J Microbiol. 2012; 2012: 358305. doi: 10.1155/2012/358305. PMID: 21977036, PMCID: PMC3185252.

6) Forbes BA, Sahm DF, Weissfeld AS, Bailey WR. Bailey and Scott's Text Book of Diagnostic Microbiology, 12th Edn. Mobsy Elsevier, St Louis, MO.

7) Venkatensan G, Singh R, Murugesan AG, Janaki C, Shankar SG. Trichophyton rubrum- the predominant etiological agent in human dermatophytoses in Chennai, India. African Journal of Microbiology Research. 52(1): 9-12.

8) Yadav A, Urhekar AD, Mane V, Singh Danu M, Goel N, Ajit KG. Optimization and Isolation of Dermatophytes from Clinical Samples and In Vitro Antifungal Susceptibility Testing By Disc Diffusion Method. Journal of Microbiology and Biotechnology. 2013; 2(3): 19-34.

9) Burzykowski T, Molenberghs G, Abeck D, Haneke E, Hay R, Katsambas A. High prevalence of foot diseases in Europe: results of the Achilles Project. Mycoses. 2003; 46(11-12): 496-505. doi: 10.1046/j.0933-7407.2003.00933.x. PMID: 14641624.

10) Theel ES, Hall L, Mandrekar J, Wengenack NL. Dermatophyte identification using matrix-assisted laser desorption ionization-time of flight mass spectrometry. J Clin Microbiol. 2011; 49(12): 4067-71. doi: 10.1128/JCM.01280-11. PMID: 21956979, PMCID: PMC3232958.

11) Matar MJ, Ostrosky-Zeichner L, Paetznick VL, Rodriguez JR, Chen E, Rex JH. Correlation between Etest, disk diffusion, and micro dilution methods for antifungal susceptibility testing of fluconazole and voriconazole. Antimicrob Agents Chemother. 2003; 47(5): 1647-51. doi: 10.1128/AAC.47.5.16471651.2003. PMID: 12709335, PMCID: PMC153338.

12) Esteban A, Abarca ML, Cabanes FJ. Comparison of disk diffusion method and broth micro dilution method for antifungal susceptibility testing of dermatophytes. Med Mycol. 2005; 43(1): 61-6. doi: 10.1080/13693780410001711972. PMID: 15712608 .

13) Pakshir K, Bahaedinie L, Rezaei $Z$, Sodaifi M, Zomorodian K. In vitro activity of six antifungal drugs against clinically important dermatophytes. Jundishapur Journal of Microbiology. 2009; 2(4): 158-63. 
14) Omar AA. Ringworm of the scalp in primary school children in Alexandria: infection and carriage. East Mediterr Health J. 2000; 6(5-6): 961-7. PMID: 12197355.

15) Achterman RR, White TC. A foot in the door for dermatophyte research. PLoS Pathog. 2012; 8(3) e1002564. doi: 10.1371/journal.ppat.1002564. PMID: 22479177, PMCID: PMC3315479.

16) Madhavi S, Rama Rao MV, Jyothsna K. Mycological study of Dermatophytosis in rural population. Annuals of Biological Research. 2011; 2(3): 88-93.

17) Sumathi S, Mariraj J, Shafiyabi S, Ramesh R, Krishna S. Clinicomycological study of dermatophytes. Int J Pharm Biomed Res. 4(2): 132-4.

18) Jha BK, Murthy SM, Devi NL. Molecular identification of dermatophytosis by polymerase chain reaction (PCR) and detection of source of infection by restricted fragment length polymorphism (RFLP). Journal of College of Medical Sciences-Nepal. 2012; 8(4): 7-15. doi: 10.3126/jcmsn.v8i4.8694.

19) Kannan P, Janaki C, Selvi GS. Prevalence of dermatophytes and other fungal agents isolated from clinical samples. Indian J Med Microbiol. 2006; 24(3): 212-15. PMID: 16912443.

20) Amer M, Taha M, Tosson Z, El-Garf A. the frequency of causative dermatophytes in Egypt. Int J Dermatol. 1981; 20(6): 431-4. doi: 10.1111/j.1365-4362.1981.tb02009.x. PMID: 7263125.

21) Emele FE, Oyeka CA. Tinea capitis among primary school children in Anambra state of Nigeria. Mycoses. 2008; 51(6): 536-41. doi: 10.1111/j.1439-0507.2008.01507.x. PMID: 18422917.

22) Nweze EI, Okafor JI. Prevalence of dermatophytic fungal infections in children: a recent study in Anambra. Mycopathologia. 2005; 160(3): 239-43. doi: 10.1007/s11046-005-0124-0. PMID: 16205973.

23) Chandra J. Textbook of Medical Mycology. Mehta Publishers, New Delhi. 1996; 67-79.

24) Rebell G, Taplin D. Dermatophytes: Their recognition and identification. 2nd ed Miami University Press, Miami. 1974; 124.

25) Mehta JP, Deodhar KP, Mehta VR, Chapnekar PM. A study of dermaotmycosis in Bombay. Indian J Pathol Microbiol. 1977; 20(1): 23-31. PMID: 873579.

26) Tampieri MP. Actuality on diagnosis of dermatomycosis. Parassitologia. 2004; 46(1-2): 183-6. PMID: 15305713.

27) Girgis SA, Zu El-Fakkar NM, Badr H, Shaker OA, Metwally FE, Bassim HH. Genotypic identification and antifungal susceptibility pattern of dermatophytes isolated from clinical specimens of dermatophytosis in Egyptian patients. Egyptian Dermatology Online Journal. 2005; 2(2).

28) Galuppi R, Gambarara A, Bonoli C, Ostanello F, Tampieri MP. Antimycotic effectiveness against dermatophytes: comparison of two in vitro tests. Vet Res Commun. 2010; 34(1): 57-61. doi: 10.1007/s11259-010-9386-1. PMID: 20490661.

29) Rezende C, Borsari GP, Da Silva AC, Cavalcanti FR. Dermatophytosis epidemiologic study in public institution of Barretos city, São Paulo, Brazil. Rev Bras Anal Clin. 40(2): 6-13.

30) Nweze EI, Mukherjee PK, Ghannoum MA. Agar-Based Disk Diffusion Assay for Susceptibility Testing of Dermatophytes. J Clin Microbiol. 2010; 48(10): 3750-2. doi: 10.1128/JCM.01357-10. PMID: 20668120, PMCID: PMC2953072.

31) Santos JI, Paula CR, Viani FC, Gambale W. Susceptibility testing of Trichophyton rubrum and Microsporum canis to three azoles by E-test. Journal de Mycologie Medicale. 2001; 11(1): 42-3. doi: 032001-11-1-1156-5233-101019.

32) Fernández-Torres B, Cabañes FJ, Carrillo-Munõz AJ, Esteban A, Inza I, Abarca L, et al. Collaborative evaluation of optimal antifungal susceptibility testing condition for dermatophytes. J Clin Microbiol. 2002; 40(11): 3999-4003. doi: 10.1128/JCM.40.11.3999-4003.2002. PMID: 12409365, PMCID: PMC139645.

33) Nweze EI, Ogbonna CC, Okafor JI. In vitro susceptibility testing of dermatophytes isolated from pediatric cases in Nigeria against five antifungals. Rev Inst Med Trop Sao Paulo. 2007; 49(5): 293-5. doi: 10.1590/S0036-46652007000500004. PMID: 18026635.

34) Magagnin CM, Stopiglia CD, Vieira FJ, Heidrich D, Machado M, Vetoratto G, et al. Antifungal susceptibility of dermatophytes isolated from patients with chronic renal failure. An Bras Dermatol. 2011; 86(4): 694-701. doi: 10.1590/S0365-05962011000400011. PMID: 21987135. 